\title{
Guías de práctica clínica del dolor neuropático (II)
}

\author{
J. Aguilera-Muñoz, E. Arizaga-Cueta, A. Carpio-Rodas, J. Crump, F. Díaz-Heredia, \\ C.F. Fernández, J.M. Griego, D. Guerrero, M. Hincapié, M.X. León, J. Moyano, \\ M. Navarro-Chávez, C.E. Rangel-Galvis, R. Rodríguez, E. Salazar-Bolaños, \\ A. Sarmiento, D. Terán Saá-Jaramillo, D. Tettamanti, D. Valencia, J.J. Vargas-Gómez
}

GUIDELINES FOR THE CLINICAL MANAGEMENT OF NEUROPATHIC PAIN (II)

\begin{abstract}
Summary. Introduction. Up to $5 \%$ of the population suffers from neuropathic pain (NP). A bibliographical search in several databases revealed that, to date, there are no protocols to guide physicians who are not specialists in pain that enable them to treat NP and thus improve patients' quality of life. Aims. The aim of this study is to provide Spanish-speaking physicians who are not specialists in pain with a set of guidelines for the treatment of NP. A bibliographical search was performed in order to base the results and conclusions on the evidence-based medicine methodology. Development. First, we review the most effective clinical and paraclinical methods for diagnosing NP, and the LANSS pain scale is reported as the most appropriate method of clinically evaluating NP. The anatomical paths and the physiology of pain are then described and we review the molecular variables involved. Finally, we point out the current therapeutic options and propose an algorithm for the treatment of NP. Conclusions. There is no specific set of guidelines for the treatment of NP. At the present time, the keystone of NP treatment consists in the use of antidepressant and anticonvulsive drugs. There is a need for further clinical trials to prove the effectiveness of using combined medication. [REV NEUROL 2005; 40: 303-16]

Key words. Clinical trials. Diagnosis. Meta-analysis. Neuropathic pain. Physiology. Therapeutics.
\end{abstract}

\section{INTRODUCCIÓN GENERAL}

Pese a los avances de las ciencias básicas y clínicas en el estudio del dolor neuropático (DN), todavía falta mucho por hacer para mejorar la calidad de vida de los pacientes afectados [1]. Por este motivo, hemos desarrollado unas guías de práctica clínica sencillas que sugieran pautas de tratamiento del DN dirigidas a los médicos que no son especialistas en dolor de habla hispana, que incluyan una comprensión fisiopatológica del síndrome y proporcionen una aproximación diagnóstica y terapéutica del problema.

El abordaje diagnóstico actual del DN hace referencia a los mecanismos que originan el dolor. Los modelos diagnósticos permiten la clasificación en cuatro grupos, de acuerdo con su mecanismo fisiopatológico:

1. Sensibilización de neuronas espinales por deaferentación.

2. Descargas ectópicas en fibras $\mathrm{C}$.

3. Sensibilización de neuronas periféricas debido a descargas ectópicas en fibras nociceptivas $\mathrm{C}$.

4. Sensibilización de neuronas espinales por la sensibilización de nociceptores $[2,3]$.

Por otro lado, la literatura no comunica descriptores patognomónicos del DN central o periférico y cada paciente puede utilizar múltiples descriptores discriminativos sensoriales para describir la afectación neurológica [4]. Un mecanismo puede ser responsable de diferentes síntomas, mientras que el mismo síntoma puede ser ocasionado por diferentes mecanismos.

\footnotetext{
Aceptado: 20.01.05.

Asociación Colombiana para el Estudio del Dolor. Bogotá, Colombia. Asociación Ecuatoriana de Endocrinología. Quito, Ecuador.

Correspondencia: Dra. Martha Ximena León. Universidad de la Sabana. Facultad de Medicina. Campus Universitario del Puente del Común. Chía, Cundinamarca, Colombia.E-mail: martha.leon@unisabana.edu.co

C 2005, REVISTA DE NEUROLOGÍA
}

El proceso diagnóstico debe incluir la historia clínica, el examen físico y los exámenes paraclínicos confirmatorios.

La historia clínica y el examen físico son todavía importantes herramientas para el diagnóstico [4], ya que no siempre los exámenes ofrecen resultados concluyentes para validar el diagnóstico [5]. Por ello, las escalas utilizadas para cuantificar los signos y síntomas del DN deben validarse previamente y tanto sus características operativas como los correspondientes exámenes paraclínicos deben valorarse a la luz de los conceptos de la medicina basada en evidencia [6].

En lo que se refiere a la terapia farmacológica y no farmacológica del DN, y con base en estudios clínicos de observación, experiencias anecdóticas y hallazgos en modelos experimentales, la terapia actual ofrece un alivio parcial del dolor en no más de la mitad de los casos. Los múltiples agentes terapéuticos, clasificados en terapias farmacológicas, técnicas de neuroestimulación e intervenciones quirúrgicas, ofrecen alivios parciales de la sintomatología. No existen evidencias de estudios de alta calidad científica que evalúen el efecto aditivo de distintas opciones terapéuticas. Además, no se han estudiado los factores pronósticos de la respuesta individual de un paciente frente a un medicamento [4]. En la práctica clínica es imperioso tener en cuenta estas limitaciones para no crear falsas expectativas al paciente. De ahí la necesidad de clasificar las mejores evidencias disponibles para plasmarlas en unas guías de DN que le sirvan al clínico en su práctica cotidiana.

A pesar de la importante controversia sobre el abordaje terapéutico, la clasificación del dolor basada en sus mecanismos moleculares permite una aproximación racional a novedosas tendencias terapéuticas que deben evaluarse rigurosamente $[2,3]$. A pesar de la evidencia disponible, varios autores consideran obligado mantener la tradicional clasificación basada en entidades diagnósticas que, de alguna forma, precedió la clasificación basada en los mecanismos de producción de dolor, con el fin de realizar un abordaje terapéutico racional [4]. Es un 
objetivo de esta guía tener en cuenta ambas aproximaciones terapéuticas y valorarlas a la luz de las evidencias científicas.

La incidencia del DN crece mundialmente $[7,8]$ como consecuencia del aumento de la expectativa de vida, la diabetes y la infección por el VIH, y a diferentes etiologías, como el cáncer, la exposición a sustancias tóxicas y los traumatismos. En la actualidad, los pacientes con DN no se diagnostican y tratan adecuada ni oportunamente [9], lo que lleva al deterioro en la calidad de vida de los mismos. La presente guía de DN ofrece una herramienta para un trabajo médico eficiente que, se espera, permita un diagnóstico oportuno y precoz y un mejor tratamiento del paciente.

\section{JUSTIFICACIÓN}

El interés predominante por desarrollar unas guías de práctica clínica es mejorar la calidad del cuidado médico de los pacientes individuales y estandarizar los tratamientos clínicos en las instituciones prestadoras de servicios de salud [10].

Antes de emprender el difícil camino de diseñar unas guías de práctica clínica de carácter regional, era relevante encontrar unas guías de práctica clínica que pudieran dar respuesta a necesidades locales y que pudieran adaptarse para su uso en el ámbito regional [11]. Tras una búsqueda sistemática, no fue posible identificar unas guías de práctica clínica de carácter integral que dieran respuesta a las necesidades regionales; esta fue la justificación primordial para el desarrollo de las presentes guías.

\section{METODOLOGÍA \\ Síntesis de las evidencias}

Las guías de recomendación están calificas para diferenciar los estudios fundamentados en una evidencia sólida, de aquellos basados en evidencias deficientes. El juicio se hace sobre una evaluación objetiva de diseño y calidad de cada estudio y, además, con base en un análisis -tal vez más subjetivo- de la consistencia, relevancia clínica y validez externa del cuerpo completo de las evidencias [12]. La calificación no guarda relación con la importancia de la recomendación, sino con la consistencia de la evidencia de sustentación y, en particular, con el poder de predicción del diseño del estudio que dio origen a la información obtenida. Así, la calificación asignada a la recomendación indica a los usuarios la probabilidad de que, si ésta se lleva a cabo, los resultados pronosticados ocurran.

Se diseñaron tablas de evidencia que compilaron la información, de manera que se resumieron todos los estudios corroborados por la investigación sistemática de la literatura que se relacionaron con cada pregunta clave. Estas tablas de evidencia representaron una importante parte del registro de desarrollo de la guía y aseguran la transparencia de la base de las recomendaciones del grupo de desarrollo de la guía.

\section{Juicio considerado}

Es sorprendentemente inusual que la evidencia muestre claramente y sin ambigüedad qué procedimiento se debería recomendar para cualquier pregunta dada. Por tanto, no resulta claro, para quienes no participaron en el proceso de la toma de decisión, cómo los desarrolladores de la guía llegaron a hacer tales recomendaciones, dada la evidencia en la que tuvieron que apoyarse. Con el fin de evitar este problema, se introdujo el concepto de juicio considerado.
Bajo el título de juicio considerado, los grupos de desarrollo de la guía resumieron su opinión del cuerpo entero de evidencias cubierto por cada tabla de evidencia. La opinión de este resumen cubrió los siguientes aspectos:

- Cantidad, calidad y consistencia de las evidencias.

- Capacidad de generalización de los resultados de los estudios.

- Capacidad de aplicación de la guía a la población blanco.

- Impacto clínico (el alcance del impacto en el paciente de la población blanco y los recursos necesarios para tratarlo).

Los grupos de desarrollo de la guía recibieron un formulario en el que registraron los puntos principales de su juicio considerado. Una vez se consideraron estos asuntos, los grupos resumieron su opinión sobre la evidencia y asignaron un nivel de evidencia antes de emitir una recomendación calificada.

\section{Niveles de evidencia y calificaciones de recomendación}

La asignación de un nivel de evidencia incluyó a todos los investigadores de un grupo o subgrupo particular (fisiopatología, diagnóstico o tratamiento) de desarrollo de la guía encargados de reseñar las evidencias referidas a cada pregunta específica. La asignación de la calificación de recomendación asociada con la pregunta incluyó a todos los miembros del grupo de desarrollo de la guía. Cuando el grupo de desarrollo de la guía no logró una recomendación unánime, se registraron formalmente las diferencias de opinión y se anotaron las razones de la discrepancia.

El sistema de calificación revisado pretendió darle mayor peso a la calidad de las evidencias que sustentan cada recomendación, y resaltar el hecho de que el cuerpo de evidencia debe considerarse como un todo, y no es adecuado basarse en un simple estudio para sustentar cada recomendación. Igualmente, pretendió dar mayor importancia a las recomendaciones sustentadas con estudios de observación de buena calidad, en los que los ensayos clínicos controlados no fueron posibles por razones prácticas o éticas. Mediante el proceso de juicio considerado, los participantes de la guía bajaron la calificación de una recomendación cuando consideraron que ésta no se podía generalizar, que no se aplicaba directamente a la población blanco, o cuando, por otras razones, se percibió la evidencia como más deficiente de lo que sugería la simple evaluación de la metodología.

\section{SISTEMA DE CALIFICACIÓN \\ Niveles de evidencia}

1++ Metaanálisis de alta calidad, revisiones sistemáticas de ensayos clínicos controlados o ensayos clínicos controlados con un riesgo de desviación muy bajo.

1+ Metaanálisis realizados adecuadamente, revisiones sistemáticas de ensayos clínicos controlados o ensayos clínicos controlados con un bajo riesgo de desviación.

1- Metaanálisis, revisiones sistemáticas de ensayos clínicos controlados o ensayos clínicos controlados con un alto riesgo de desviación.

$2++$ Revisiones sistemáticas de alta calidad de control de casos o cohorte, o estudios de observación.

Estudios de alta calidad de control de casos o cohorte con un muy bajo riesgo de confusión, desviación, o posibilidad y una alta probabilidad de que la relación sea casual.

2+ Estudios de control de casos o de cohorte adecuadamente 
conducidos con un bajo riesgo de confusión, desviación, o posibilidad y una moderada probabilidad de que la relación sea casual.

2- Estudios de control de casos o de cohorte con un alto riesgo de confusión, desviación, o posibilidad y un riesgo significante de que la relación no sea casual.

3 Estudios no analíticos, como informes o series de casos.

4 Opiniones de expertos.

\section{Calificaciones de recomendación}

A. Por lo menos, un metaanálisis, una revisión sistemática o ensayos clínicos controlados calificados con $1++$, y directamente aplicables a la población blanco; o una revisión sistemática de ensayos clínicos controlados o un cuerpo de evidencias compuesto principalmente por estudios calificados con $1+$, directamente aplicables a la población blanco y que demuestren la consistencia general de los resultados.

B. Un cuerpo de evidencias que incluya estudios calificados con $2++$, directamente aplicables a la población blanco y que demuestren la consistencia general de los resultados; o evidencia extrapolada de estudios calificados con $1+$ o $1+$.

C. Un cuerpo de evidencias que incluya estudios calificados con $2+$, directamente aplicables a la población blanco y que demuestren la consistencia general de los resultados; o evidencia extrapolada de estudios calificados con $2++$.

D. Nivel de evidencia 3 o 4; o evidencia extrapolada de estudios calificados con $2+$.

\section{Puntos de práctica adecuados}

Son la mejor práctica recomendada con base en la experiencia clínica del grupo de desarrollo de la guía.

A veces, los grupos de desarrollo de la guía consideran que existe un importante punto práctico que les gustaría destacar, pero para el cual no hay, y tampoco creen vaya a existir, ninguna evidencia de investigación. Esto sucederá cuando algún aspecto del tratamiento se considere como una práctica clínica tan acertada que nadie se atreva a cuestionarla. A esto se le ha dado el nombre de puntos de práctica adecuados en la guía, y se les identifica con un signo $\checkmark$. Debe quedar claro que éstos no constituyen una alternativa a las recomendaciones basadas en la evidencia, y que sólo deberían emplearse cuando no existan otros medios para abordar el asunto.

\section{GRUPO INVESTIGADOR}

El presente grupo investigador está constituido, según recomiendan las pautas de medicina basada en evidencia [11,13], por:

- Profesionales especialistas en el tratamiento del DN desde un punto de vista multidisciplinario: anestesiólogos, fisiatras, neurólogos, endocrinólogos.

- Un profesional especialista en epidemiología clínica y con entrenamiento en farmacoeconomía, encargado de asesorar sobre la metodología y estandarizar la adecuada clasificación de las evidencias.

- Un consultor de la industria farmacéutica encargado de liderar al grupo en el área logística y motivacional.

- Profesionales en bibliotecología, especialistas en búsqueda sistemática de literatura biomédica.

- Profesionales en bioestadística.

- Un médico y periodista entrenado en publicaciones médicas.

- Técnicos en digitalización y edición de literatura biomédica.
Todos los profesionales firmaron un contrato de prestación de servicios con Pfizer, empresa que se comprometió a patrocinar los gastos de todo orden necesarios para el desarrollo y ejecución de la investigación. Por la naturaleza de la investigación, cada uno de los participantes se comprometió a desarrollarla con total autonomía científica e independencia en sus resultados, y de esa forma fue entendido y aceptado por la empresa patrocinadora.

Una vez que se identificaron los artículos, se evaluaron para ver si cumplían los criterios de inclusión para la revisión. La guía tuvo en cuenta las siguientes pautas metodológicas:

- Más de un revisor evaluó la relevancia de cada artículo. Las decisiones relacionadas con la inclusión de los artículos las tomaron especialistas en DN.

- Los revisores que evaluaron la relevancia de los estudios tuvieron acceso a los nombres de los autores sus instituciones y las revistas donde se publicaron los estudios y resultados después de aplicados los criterios de inclusión y tuvieron un entrenamiento que estandarizó la evaluación de los artículos.

- Más de un revisor aplicó los criterios de inclusión a todos los artículos evaluados y potencialmente relevantes. Se contó con revisores expertos en $\mathrm{DN}$ y con profesionales no expertos que revisaron independientemente el documento.

- Las discrepancias sobre si un ensayo se incluía o no en la revisión se resolvieron mediante discusión. Cuando la discrepancia se debió a una diferencia de interpretación, el tema se resolvió por consenso.

- Se realizó un entrenamiento específico y una prueba piloto sobre la aplicación de los criterios de inclusión a una muestra de artículos.

- Se usaron métodos no formales de consenso para desarrollar recomendaciones en áreas donde no existía evidencia disponible. Este proceso identificó preguntas de investigación que no se han contestado [14].

Los médicos, en la actualidad, necesitan guías de práctica clínica fáciles de usar, sencillas y específicas. La presente guía pretendió definir las decisiones más relevantes a las que pueda enfrentarse un clínico frente a un paciente específico y determinar las posibles consecuencias de dicha decisiones. Fue necesario limitar las guías para que fueran útiles para el clínico no especialista en DN [13].

La presente guía tuvo en cuenta aspectos relevantes del área fisiopatológica, con el fin de abordar un campo comprensible en al área de diagnóstico y tratamiento. En lo posible, se diseñaron diagramas de flujo y algoritmos en la etapa de digitalización, una vez que se termino el proceso de investigación. La información se presentó en un formato sencillo y flexible, de forma que pueda aplicarse a pacientes y circunstancias específicas [13].

\section{CONFLICTOS DE INTERÉS}

La revisión estuvo, en lo posible, libre de cualquier sesgo real o potencial causado por la recepción de beneficio en efectivo o en especies de derivado de la fuente de financiación [15]. Los profesionales participantes firmaron un contrato de prestación de servicios con Pfizer, empresa que se comprometió a patrocinar los gastos de todo orden necesarios para el desarrollo y la ejecución de la presente investigación. Cada uno de los investigadores se comprometió a desarrollarla con total autonomía científica e independencia en sus resultados, y de esa forma fue entendido y aceptado por la empresa patrocinadora. 


\section{RESULTADOS}

\section{Fisiopatología}

\section{Volumen y cuerpo de la evidencia}

La revisión de la literatura incluyó artículos desde 1999 hasta 2002. De 194 artículos encontrados en la literatura mundial, se preseleccionaron 42 (por el título y el resumen estructurado), que se evaluaron según la metodología; finalmente, se seleccionaron 11.

Dos artículos fueron seleccionados unánimemente por el grupo investigador por su pertinencia y aplicabilidad.

Los artículos corresponden a revisiones no sistemáticas de la literatura y opiniones de expertos. El cuerpo de la evidencia lo constituyen los trabajos de Sandkuhler et al [16] y Giamberardino [17].

\section{Aplicabilidad}

El contenido del cuerpo de la evidencia en fisiopatología es el punto de partida para la comprensión de los procesos de diagnóstico y tratamiento de DN; por eso se tuvo en cuenta en la revisión.

La metodología de evaluación de los niveles de evidencia no se aplicó para el área de fisiopatología. La forma de obtener el conocimiento en fisiopatología se basa en estudios diferentes a los reconocidos por la metodología seguida en el desarrollo de la guía.

\section{Consistencia}

Los hallazgos de los 13 artículos son consistentes en la caracterización de la fisiopatología del DN. Los más relevantes, y que se discuten con detenimiento, son los siguientes:

- Sensibilización periférica y central.

- Desinhibición de los mecanismos moduladores del dolor.

- Reconexiones y reorganización neuronal.

- Descargas ectópicas espontáneas y evocadas.

\section{Impacto clínico}

Definen y señalan las alteraciones del sistema nervioso central (SNC) y periférico (SNP), desde los mecanismos que generan el DN, que contribuyen a un enfoque racional del tratamiento.

\section{Nivel de evidencia y grados de recomendación}

No se aplican.

\section{Diagnóstico \\ Volumen y cuerpo de la evidencia}

El volumen de la evidencia lo constituyen 160 artículos de revisión de la literatura, lo que representa el 51\% del total de la búsqueda. Los investigadores definieron la pertinencia de los artículos con base en los títulos de los artículos y en sus correspondientes resúmenes estructurados, y se obtuvieron 59.

En los 59 encontrados se aplicó la lista de chequeo para verificar la calidad metodológica.

El cuerpo de la evidencia incluyó siete artículos, de los cuales cinco tienen muy buena calidad metodológica y dos son consistentes con el cuerpo de la evidencia. Los restantes se excluyeron por no cumplir criterios de inclusión.

\section{Consistencia}

Hay una gran consistencia en los pocos artículos (siete) del cuerpo de la evidencia; todos se consideran como referencias para el diagnóstico del DN: la historia clínica y el examen físico.

\section{Impacto clínico}

En la actualidad, los pacientes con DN no se diagnostican ni adecuada ni oportunamente, lo cual lleva al deterioro de su calidad de vida. El impacto del desarrollo de estas guías permitirá un diagnóstico oportuno y precoz que resultará en un mejor tratamiento del paciente y ofrecerá una herramienta para un trabajo médico eficiente.

Los estudios de LANSS [5] y Backonja [19] son estudios clínicos controlados enfocados al diagnóstico con poco sesgo que se aplicaron a una población de 60 y 528 pacientes, respectivamente. Los dos estudios mencionados se realizaron adecuadamente para minimizar los sesgos.

Niveles de evidencia $1+$

Recomendación B.

\section{Tratamiento}

\section{Volumen y cuerpo de la evidencia}

Después de tres cribados, se analizaron 300 artículos. Incluían ensayos clínicos controlados, revisiones sistemáticas no metaanalíticas y metaanálisis realizados en adultos con DN. Se tomó en consideración lo siguiente:

- Calidad metodológica que se tuvo en cuenta.

- Asignación aleatoria y ciega de los pacientes.

- Tamaño de la muestra, magnitud del efecto y significación estadística.

- Medidas de impacto que se tuvieron en cuenta (desenlaces).

- Aplicación de escalas: de actividad física, funcional, evaluación del dolor, estado mental y depresión.

- Habilidad para manejar y/o controlar el dolor.

- Niveles subjetivos de dolor.

- Control del dolor.

- Reactivación del dolor.

- Efectos adversos relacionados con el tratamiento.

El cuerpo de la evidencia lo constituyen 35 artículos, que se clasificaron como sigue: opiáceos (12 artículos), antidepresivos (3), anticonvulsionantes (9), lidocaína (3), ketamina (3) y otros (5): pancreocimina, diclofenaco, clonidina y nemantine.

\section{Aplicabilidad}

La aplicabilidad es relativa, debido a la heterogeneidad del síndrome y de las revisiones sistemáticas encontradas. No se definen los tipos de patología y las características del dolor. Esta evidencia es independiente de los mecanismos fisiopatológicos del dolor y de la evolución de la entidad (enfermedad) concomitante o desencadenante.

La toma de decisiones para la aplicación de un tratamiento debe basarse en la efectividad y la inocuidad. Los resultados encontrados son específicos en variables como efectividad y efectos adversos; sin embargo, las dosis para manejar cada patología no están apoyadas con evidencias disponibles.

\section{Consistencia}

En los estudios individuales, el intervalo de consistencia es débil. Ésta se fortalece en el análisis sistemático, bien de revisiones sistemáticas metaanalíticas o de no metaanalíticas.

\section{Impacto clínico}

El impacto es positivo. Si bien el DN es todavía un problema, la 
Tabla I. Etiología del dolor neuropático [23].

Dolor neuropático periférico

\begin{tabular}{l}
\hline Neuropatía diabética \\
\hline Dolor posherpético \\
\hline Neuropatías por atrapamiento (síndrome del túnel carpiano) \\
\hline Polirradiculoneuropatía (cervical, dorsal, lumbar o sacra) \\
\hline Neuralgias yatrogénicas (posmastectomía, postoracotomía) \\
\hline Peuralgia del trigémino \\
\hline Polineuropatía alcohólica
\end{tabular}

Síndrome regional complejo

Neuropatía por VIH

Por compresión o infiltración tumoral

Por deficiencia nutricional

Dolor del miembro fantasma

Plexopatía postirradiación

Postraumático

Postictus

Dolor neuropático central

Mielopatía compresiva por estenosis medular

Mielopatía por VIH

Esclerosis múltiple

Enfermedad de Parkinson

Mielopatía postisquémica

Mielopatía posradiación

Postraumatismo con lesión del cordón espinal

Siringomielia

Enfermedad cerebrovascular

evidencia apoya que, a través del tiempo, algunos medicamentos demuestran su mayor eficacia e inocuidad con respecto a otros.

\section{Niveles de evidencia $1+$}

Recomendación B.

\section{CONCLUSIONES}

El DN que se genera por una lesión primaria o por una disfunción transitoria en el SNC y/o el SNP $[5,18,20]$ es el resultado de múltiples etiologías. Clínicamente, exhibe variados síndromes. Puede considerarse como una categoría de dolor con múltiples subtipos [20].

La sospecha clínica es el primer paso para la detección temprana de los pacientes con factores de riesgo algogénico y/o que padecen DN consecuente o primario. El diagnóstico del DN se fundamenta inicialmente en descripciones verbales proporcio- nadas por el paciente, que se suman a los hallazgos en el examen físico [5,19,21]. Las implicaciones terapéuticas del DN requieren aproximaciones clínicas que permitan diferenciarlo del dolor nociceptivo [9,22].

Los factores de riesgo algogénicos son:

- Causas de DN (Tabla I).

- Síndromes clínicos de DN.

\section{Aproximación diagnóstica}

En la literatura médica no hay datos suficientes que permitan hacer una recomendación de tipo A específica para el diagnóstico de DN. Tampoco existen escalas o cuestionarios validados en español que permitan su aplicación en los países de habla hispana.

Las escalas son herramientas que permiten una aproximación sistemática y unificada para el diagnóstico y el control clínico de los pacientes. En referencia al DN, existen tres escalas validadas: dos en inglés (LANSS [5] y Backonja [19]) y una en francés, de Boureau et al [23], escalas que coinciden parcialmente en sus contenidos.

Recientemente, Bennett desarrolló la escala de dolor LANSS (del inglés, the Leeds Assessment of Neuropathic Symptoms and Signs) para la evaluación de la alodinia y las alteraciones en el umbral del dolor. Puede aplicarse de forma rápida en la cabecera del paciente. En opinión de los autores, la escala de LANSS ofrece ventajas y un evidencia sólida por su fácil aplicación y simplicidad. Está pendiente su validación definitiva al idioma español (comunicación personal con el autor) [5].

El diagnóstico de DN es esencialmente clínico. Las bases se fundamentan en la historia clínica y en el examen físico del paciente [24-26]. Los exámenes de laboratorio, de imagen y de electrofisiología confirman el diagnóstico clínico, pero los resultados negativos no excluyen el diagnóstico de DN [27-32].

\section{Historia clínica}

Motivo de consulta y enfermedad actual

Frente al DN, el médico debe investigar sus características. Éstas pueden ser las siguientes:

- Tiempo de evolución.

- Patrón de duración (si es paroxístico, intermitente, permanente, continuo).

- Cualidad (punzante, quemante o corrientazo).

- Localización: el paciente debe dibujarlas (Figs. 1 y 2).

- Intensidad: el paciente debe describir la intensidad de su dolor. Existen escalas donde la persona describe la intensidad subjetiva de su dolor en una escala de 0 a 10 , donde 0 (cero) indica que no hay dolor y 10 (diez) expresa el máximo dolor que se pueda sentir. También puede indicar sobre una línea que mide $100 \mathrm{~mm}$ el grado de intensidad del dolor.

- Causa: se debe enfatizar en la etiología; las causas pueden ser infecciosas, tumorales, traumáticas, metabólicas o relacionada con alguna enfermedad (p. ej., diabetes).

\section{Examen físico}

El examen físico general es fundamental para la búsqueda de algún signo o enfermedad sistémica que oriente el diagnóstico de DN y sus posibles etiologías. Además, debe realizarse una valoración neurológica. La valoración neurológica comprende los siguientes aspectos:

- Valoración del sistema nervioso sensitivo. Con énfasis en las zonas que el paciente describe como dolorosas. Debe explorarse la alodinia (Tabla II) y la hiperalgesia (Tabla III) 


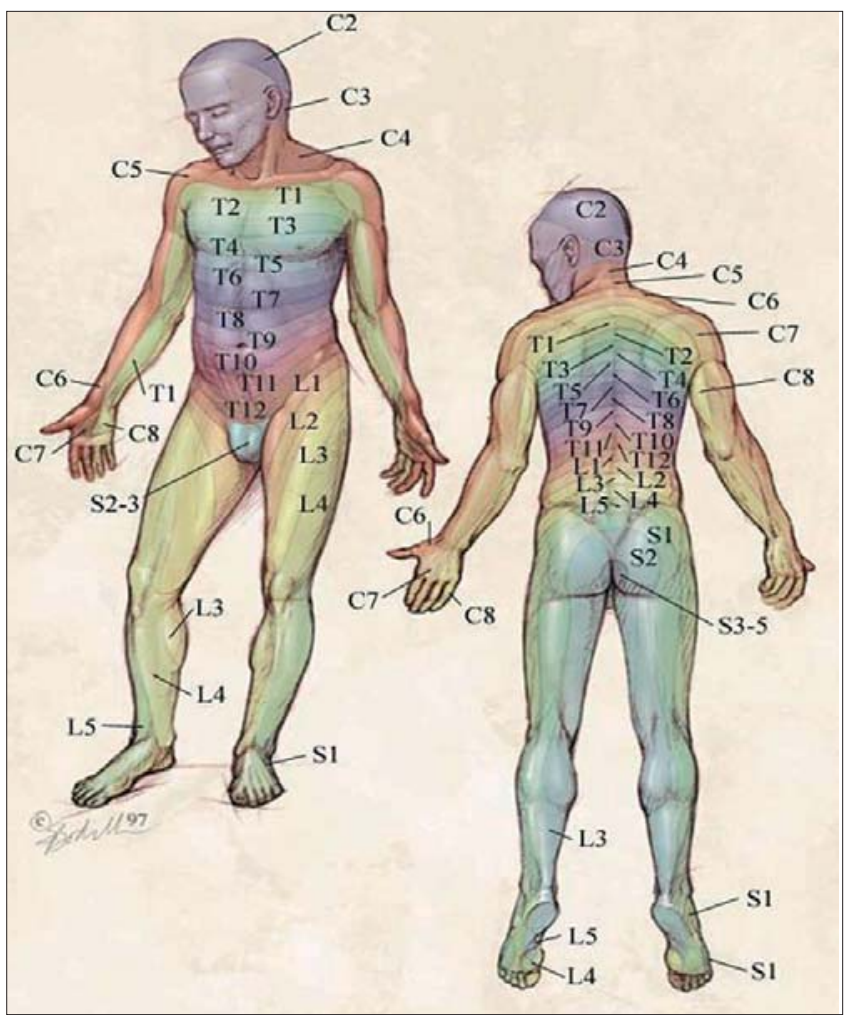

Figura 1. Dermatomas (tomado de Pitkin G. Conduction anesthesia anatomy of the spinal nerve. Philadelphia: Lippincott. p. 172-8).

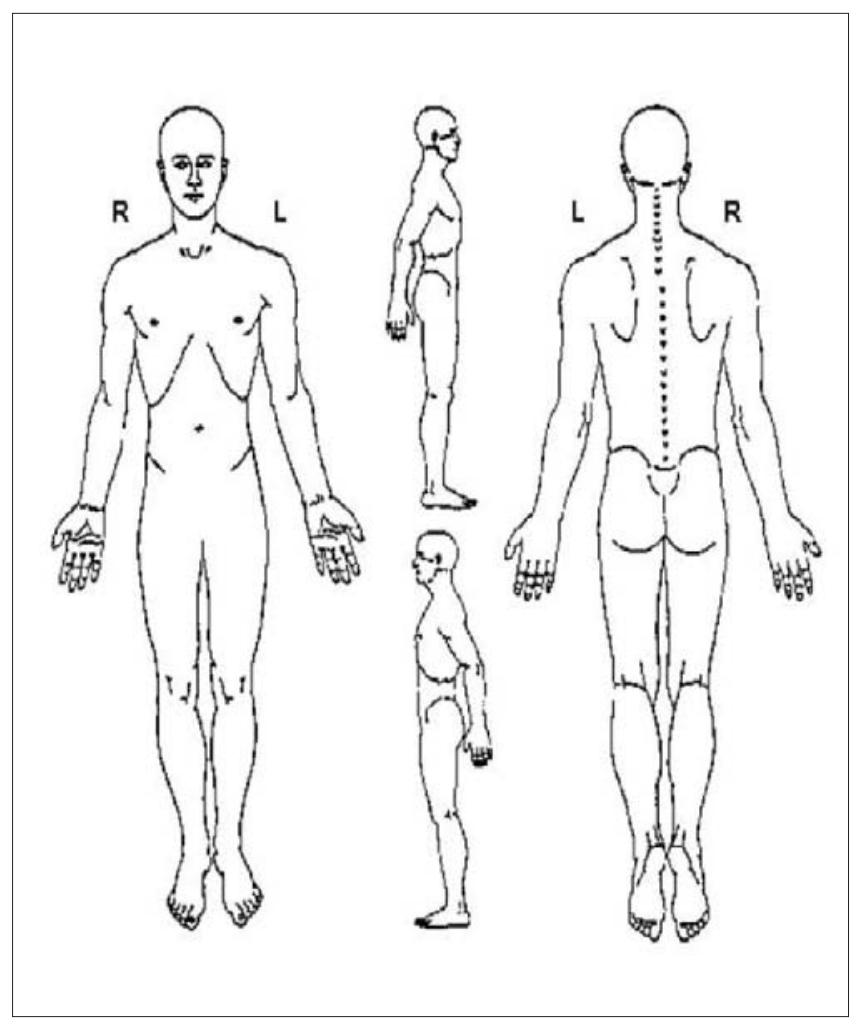

Figura 2. Mapa del dolor (tomado de Pitkin G. Conduction anesthesia anatomy of the spinal nerve. Philadelphia: Lippincott. p. 172-8). con estímulos mecánicos (presión, tacto, pinchazo) o estímulos térmicos (frío, calor). Debe explorarse la propiocepción y la percepción de estímulos vibratorios con un diapasón de 128 cps (glosario al final de la guía). El DN puede seguir patrones segmentarios; esto, por afectación de una raíz (dermatoma o de un nervio especifico). Por ejemplo, en el caso de la diabetes, el dolor puede describirse como dolor en forma de guante o de bota debido a la afectación de nervios distalmente.

- Valoración del sistema nervioso motor. Se debe evaluar la fuerza segmentaria (patrones de inervación), el tono, el trofismo muscular, los movimientos anormales, las posiciones antálgicas y la disfunción de los movimientos activos y pasivos.

La escala de fuerza muscular es la siguiente: 0 , sin movimiento; 1 , indicios de movimiento activo; 2, movimiento activo en ausencia de gravedad; 3, movimiento activo contra la gravedad, en ausencia de resistencia; 4, movimiento activo capaz de vencer la gravedad y cierta resistencia, y 5 , movimiento activo de fuerza normal.

El área motora afectada se relaciona con los segmentos neurológicos: cuello y hombro, $\mathrm{C} 1-\mathrm{C} 4$; brazo, mano y dedos,
Tabla II. Exploración de la alodinia.

\begin{tabular}{lll}
\hline Tipo de exploración & Valoración & Respuesta esperada \\
\hline Mecánica & Ligera presión manual sobre la piel & Dolor lento \\
\hline Mecánica estática & Ligero pinchazo con objeto punzante & Dolor cortante superficial \\
\hline Mecánica dinámica & Roce de la piel con un algodón & $\begin{array}{l}\text { Dolor cortante, quemante, } \\
\text { superficial }\end{array}$ \\
\hline Térmica al calor & Tocar la piel con objetos a $40^{\circ} \mathrm{C}$ & \begin{tabular}{l} 
Sensación quemante dolorosa \\
\hline Térmica al frío
\end{tabular} \\
\hline
\end{tabular}

Tabla III. Exploración de la hiperalgesia.

\begin{tabular}{lll}
\hline Tipo de exploración & Valoración & Respuesta esperada \\
\hline Mecánica estática & Ligero pinchazo con objeto punzante & Dolor lento superficial \\
\hline Térmica al frío & Contacto de la piel con alcohol & Sensación quemante dolorosa \\
\hline Térmica al calor & Tocar la piel con un objeto a $45^{\circ} \mathrm{C}$ & Sensación quemante dolorosa \\
\hline
\end{tabular}




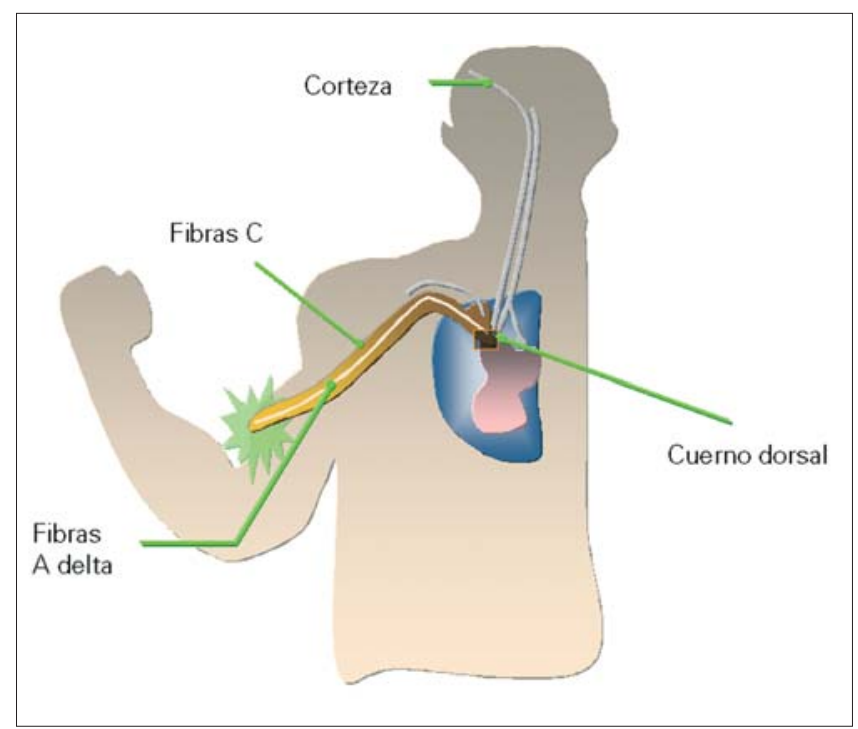

Figura 3. Anatomía del dolor. La información dolorosa captada por los nociceptores en las terminales nerviosas libres es trasmitida por la primera neurona, que hace sinapsis en el cuerno dorsal de la médula espinal con la segunda neurona, la cual se proyecta a la corteza cerebral y hace relevo en el tálamo. Las fibras C Ilegan a las láminas I y II de Rexed en el cuerno dorsal, mientras las fibras A- $\beta$ Ilegan a las láminas III y IV.

Tabla IV. Exámenes paraclínicos indicados para algunas patologías que concursan con dolor neuropático.

\begin{tabular}{ll}
\hline Patología & Exámenes paraclínicos \\
\hline $\begin{array}{l}\text { Dolor mantenido por simpático } \\
\text { Síndrome regional complejo }\end{array}$ & $\begin{array}{l}\text { Bloqueos simpáticos [36] } \\
\text { (confirma el diagnóstico) } \\
\text { Termografía } \\
\text { Test de Minor }\end{array}$ \\
\hline Síndrome de túnel del carpo & Velocidades de conducción \\
& motora y sensitiva \\
& Electromiografía \\
\hline Síndrome compartimental & Electromiogras evocados [37-40] \\
de la extremidad inferior & Exámenes neurofisiológicos: \\
& - Electroneurografía \\
& - Exámenes cuantitativos sensoriales \\
\hline Diabetes & Velocidades de conducción \\
& nerviosa sensitiva y motora [42] \\
& Termografía \\
\hline Dolor lumbar crónico & Electromiografía \\
\hline
\end{tabular}

C6; tríceps, C7-C8; rotuliano, L2-L4; aquilino, L5-S1, y plantar, L4-L5, S1-S2.

- Valoración del sistema nervioso autónomo. Se evalúa: temperatura, regulación vasomotora (cambios de tensión arterial con la postura), sudoración, reacciones pilomotoras, cambios tróficos (en pelo, uñas y piel) y otras anomalías (por ejemplo, edema periférico neuropático).

\section{Análisis diagnósticos complementarios}

El diagnóstico de DN puede confirmarse mediante algunos exámenes complementarios. Entre ellos, pueden citarse los siguientes:
- Estudios de conducción nerviosa: cualifican la velocidad de conducción sensitiva y motora y definen la magnitud de las fibras afectadas.

- Microneurografía: determina la presencia de impulsos ectópicos que se originan en las fibras sensoriales.

- Electromiografía: cualifica las características intrínsecas del músculo en un área dolorosa.

- Prueba de Minor: se utiliza para detectar alteraciones en la sudación.

- Pruebas autonómicas cardiovasculares.

- Instrumentos: aunque escasos, existen algunos elementos que en el examen físico evocan una respuesta dolorosa; entre ellos, se pueden citar:

- Algómetro: es un elemento que mide la presión a la cual un punto del cuerpo se torna doloroso.

- Prueba térmica: mide los umbrales para el estímulo de temperatura.

- Cabellos de von Frey: miden el umbral para el estímulo mecánico.

- Vibrámetro: mide el umbral para la percepción de la vibración.

La tabla IV presenta algunos exámenes complementarios que pueden usarse como apoyo diagnóstico en algunas patologías que dan lugar a DN.

\section{FISIOPATOLOGÍA \\ Generalidades}

El DN es aquel generado por lesión primaria o por disfunción del SNC o el SNP, como una reacción de mala adaptación que altera el equilibrio normal entre los impulsos dolorosos y los no dolorosos [43,44].

El DN puede ser causado por diversas etiologías, como metabólicas (diabetes), isquémicas (infarto cerebral), compresivas (radiculopatías o neuralgia del trigémino), infecciosas (HIV, herpes zoster), traumáticas (lesión de la médula espinal), tóxicas (plomo), autoinmunitarias, neoplásicas y hereditarias [45] (Tabla I).

\section{Anatomía del dolor}

Las terminaciones nerviosas libres se distribuyen en los tejidos cutáneos, musculares, articulares y viscerales; son, en esencia, receptores del dolor (nociceptores) y se clasifican como polimodales, mecánicos y térmicos. Los receptores polimodales captan estímulos diversos, los cuales son transmitidos por fibras $\mathrm{C}$ delgadas no mielinizadas a una velocidad de conducción lenta, no mayor de $1-2 \mathrm{~m} / \mathrm{s}$; además, son receptores con un umbral alto. Los nociceptores mecánicos y térmicos captan estímulos específicos de presión, posición y temperatura que se transmiten por fibras A- $\delta$ de escasa mielinización y una velocidad de conducción de 6-30 m/s [46-50].

La información dolorosa que viaja por estas fibras llega hasta los somas de sus neuronas, localizados en los ganglios de las raíces dorsales paraespinales y del nervio trigémino. Ésta es la primera neurona aferente, y hace sinapsis mediante su proyección axonal central con la segunda neurona, situada en las láminas I y II de Rexed del asta dorsal de la médula espinal. Estas láminas están formadas por neuronas que reciben aferencias específicas de dolor. Las capas más profundas (III y IV) reciben axones de fibras A- $\beta$ con información sensorial propioceptiva de posición, movimiento y tacto fino. Bajo condiciones normales, no reciben información dolorosa [46,48,50] (Fig. 3). 


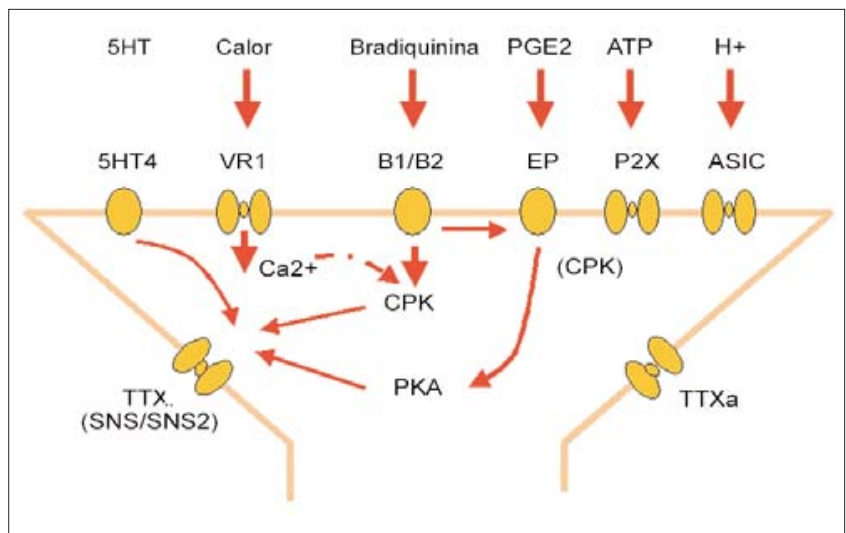

Figura 4. Sensibilización periférica. El estímulo constante de los receptores respectivos por sustancias como la serotonina, las prostaglandinas, la bradicinina y la histamina como parte la inflamación neurogénica conlleva al aumento del calcio intracelular y la activación de las cinasas.

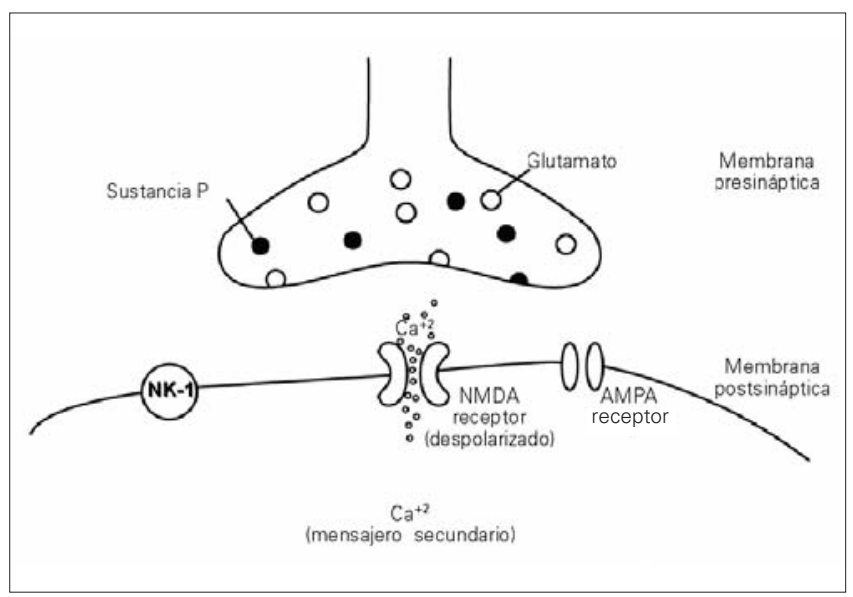

Figura 6. Sensibilización central. La liberación sostenida de glutamato, sustancia $P$ y neurocinas por las fibras $C$ activa los receptores AMPA y NMDA, retira permanentemente el magnesio, lo que permite la entrada masiva de calcio a la segunda neurona y aumenta su exitabilidad. También actúa como segundo mensajero e induce una serie de cambios duraderos en las neuronas del asta dorsal.

Los axones de las neuronas de segundo orden cruzan en su mayoría por delante de la comisura anterior, para luego ascender por el tracto espinotalámico lateral hasta los núcleos posterolateral, ventral y medial del tálamo, donde hacen relevo con las neuronas de tercer orden, que proyectan a la corteza cerebral del lóbulo parietal, área somatosensorial, donde el dolor se hace consciente. El estímulo doloroso también asciende por una vía alterna contralateral desde el asta dorsal, pasa por el bulbo, los núcleos parabraquiales del puente, los núcleos intralaminares del tálamo y el hipotálamo, para finalizar en amplias áreas de la corteza límbica, cingulada e insular [46,50]. Estas estructuras permiten la participación de los componentes autonómicos, endocrinos, emocionales y de memoria, los cuales se integran y generan un proceso inverso (descendente) de modulación inhibitoria del dolor, que termina en el asta dorsal de la médula espinal. El objetivo de la modulación descendente es contrarrestar la intensidad del estímulo doloroso. En este proceso se sintetizan y liberan opioides endógenos, serotonina, monoaminas y algunas hormonas. Cuando el estímulo supera en intensidad estos mecanismos protectores, el dolor asciende fácilmente a niveles superiores [50].

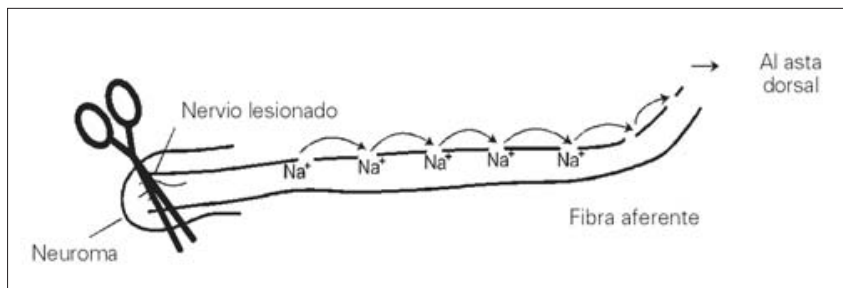

Figura 5. Generación de descargas ectópicas. La lesión del nervio distalmente induce cambios genotípicos funcionales que modifican la expresión de los canales de sodio dependientes de voltaje, los cuales, a su vez, incrementan la propagación de los estímulos.

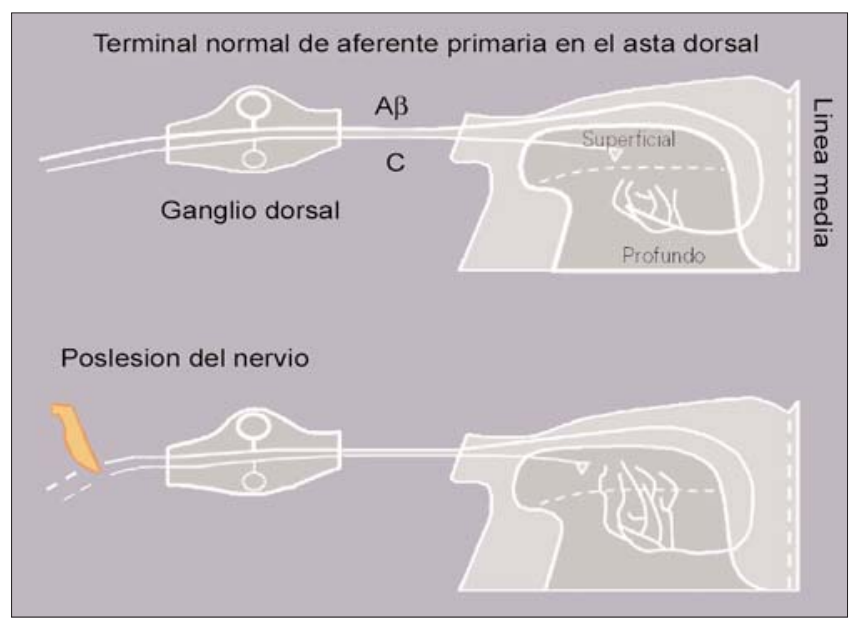

Figura 7. Reorganización central de fibras. La lesión del nervio periférico, por procesos de neuroplasticidad lleva a la propagación de las fibras A- $\beta$ que normalmente terminan en las láminas III y IV de Rexed, a que invadan la lámina II y hagan que estímulos normalmente no dolorosos se perciban como dolorosos.

Tabla V. Correlación de síntomas, signos y mecanismos de dolor neuropático descritos.

\begin{tabular}{lll}
\hline Síntoma-signo & Mecanismo & $\begin{array}{l}\text { Estructura-vía- } \\
\text { neurotransmisores }\end{array}$ \\
\hline $\begin{array}{l}\text { Hiperalgesia } \\
\text { (mecánica estática y } \\
\text { térmica); dolor espontáneo }\end{array}$ & $\begin{array}{l}\text { Sensibilización } \\
\text { periférica }\end{array}$ & $\begin{array}{l}\text { Receptores vaniloides } \\
\text { Bradicinina y serotonina }\end{array}$ \\
\hline $\begin{array}{l}\text { Dolor espontáneo (lancinante, } \\
\text { quemante y choque eléctrico) }\end{array}$ & $\begin{array}{l}\text { Descargas } \\
\text { ectópicas }\end{array}$ & $\begin{array}{l}\text { Canales de sodio } \\
\text { y potasio }\end{array}$ \\
\hline $\begin{array}{l}\text { Dolores espontáneos } \\
\text { Conexiones } \\
\text { efápticas }\end{array}$ & $\alpha-1$ \\
\hline $\begin{array}{l}\text { Hiperalgesia y dolor } \\
\text { espontáneo }\end{array}$ & $\begin{array}{l}\text { Desinhibición } \\
\text { central }\end{array}$ & $\begin{array}{l}\alpha-2, \text { GABA, adenosina, } \\
\text { canales de sodio, CCK }\end{array}$ \\
\hline $\begin{array}{l}\text { Hiperalgesia táctil dinámica; } \\
\text { dolor como pinchazo }\end{array}$ & $\begin{array}{l}\text { Sensibilización } \\
\text { central }\end{array}$ & $\begin{array}{l}\text { NMDA, Cox-2, NK1, } \\
\text { PKC, MAPK }\end{array}$ \\
\hline
\end{tabular}

Las vías descendentes de la modulación inhibitoria del dolor se originan en la sustancia gris periacueductal, en el núcleo ventral segmental mesencefálico (opioides endógenos), en el locus ceruleus (monoaminas) y en los núcleos del rafe medio del bulbo (serotonina), y se integran en el funículo dorsolateral multisináptico descendente [47,50].

La vía neurofisiológica del dolor se mantiene por los permanentes estímulos neurogénicos, tanto periféricos como centrales, así como de sus vías aferentes y eferentes, y constituye es- 
Tabla VI. Niveles de evidencia de los antidepresivos.

\begin{tabular}{|c|c|c|c|}
\hline Autor & Objetivo & Calificación & Observaciones \\
\hline $\begin{array}{l}\text { McQuay HJ, } \\
\text { (metaanálisis) } \\
\text { [59] }\end{array}$ & $\begin{array}{l}\text { Revisión sistemática de } \\
\text { antidepresivos en dolor } \\
\text { neuropático: nortriptilina, } \\
\text { imipramina, desipramina, } \\
\text { fluoxetina, clomipramina, } \\
\text { paroxetina y amitriptilina }\end{array}$ & $\begin{array}{l}\text { Evidencia } 1+ \\
\text { Recomendación B }\end{array}$ & $\begin{array}{l}\text { Neuropatía diabética RR: } 3,6(2,5-5,2) \text { NNT: } 3 \\
\text { Neuralgia postherpética RR: 6,8 (3,5-14,3) NNT: } 2,3 \\
\text { Dolor facial atípico 4,1 RR: }(2,3-7,5) \text { NNT 2,8 } \\
\text { No existió diferencia significativa entre los } \\
\text { antidepresivos incluidos } \\
\text { Eventos adversos menores NNH 3,7 }(2,9-5,2) \\
\text { Efectos adversos mayores NNH } 22(13,5-58) \text {. }\end{array}$ \\
\hline
\end{tabular}

produce una serie de cambios histoquímicos humorales y vasogénicos que pueden perpetuarse aun después de terminada ésta. Esto produce una estimulación repetitiva del nociceptor en el sitio de la lesión que perdura [49].

Los cambios humorales
Tabla VII. Revisión sistemática Colaboración Cochrane [58].

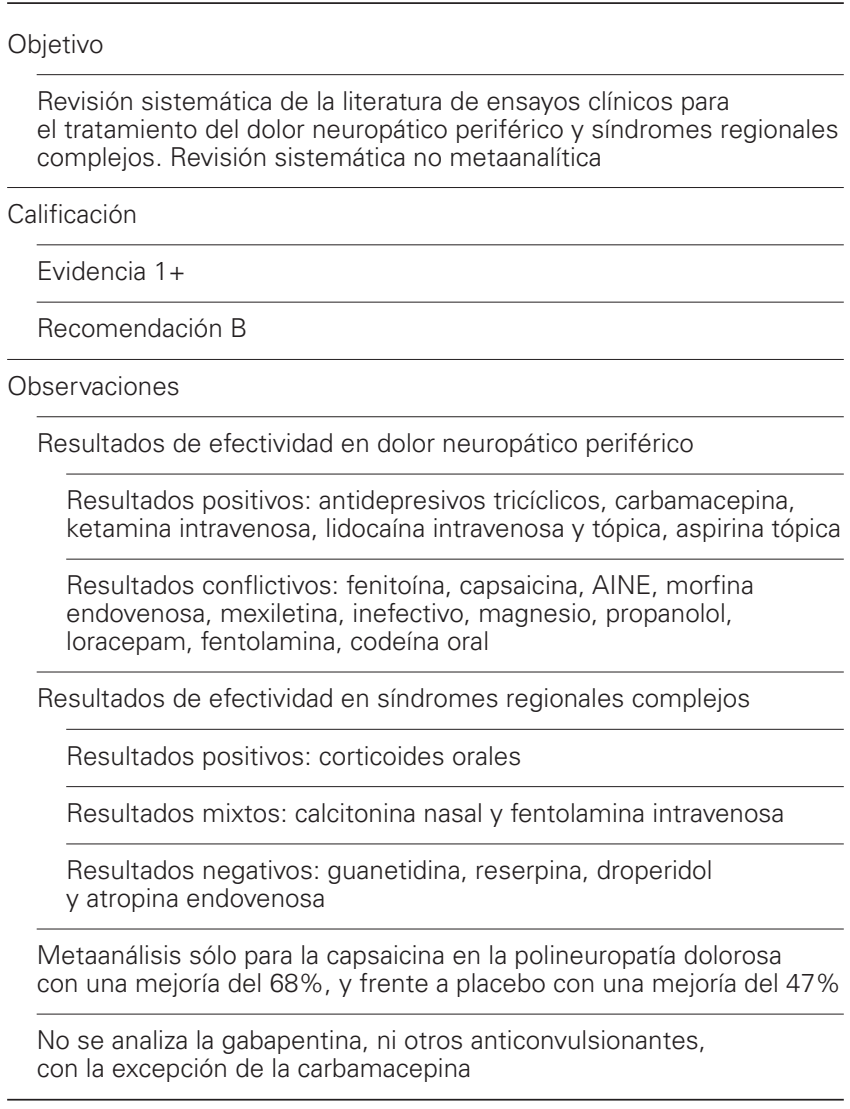

tructuras anatomofuncionales que producen sustancias neurotróficas, como el GDNF (factor neurotrófico derivado de la glia) y el NGF (factor de crecimiento nervioso), que garantizan la supervivencia, vitalidad y crecimiento de las neuronas de este sistema $[48,49]$.

\section{Neurobiología del dolor neuropático}

Cualquier agresión contra el organismo, por ejemplo, una lesión inflamatoria, genera como mecanismo de defensa un dolor agudo nociceptivo que se resuelve al terminar el estímulo. Su persistencia no tiene justificación. En ocasiones, una lesión o disfunción del sistema nervioso causa un dolor sostenido en el tiempo y que desborda la causa que lo generó, sin ningún efecto protector o benéfico para el organismo [48,49].

\section{Mecanismos del dolor neuropático Sensibilización periférica}

La sensibilización periférica es la primera fase de la afectación neuropática, y como respuesta inflamatoria ante una agresión se hacen referencia al incremento en el tejido inflamado de sustancias como prostaglandinas, serotonina, bradicinina, adenosina y adrenalina, así como el NGF, entre otros, que actúan sobre receptores específicos. Como consecuencia de lo anterior, hay un aumento intracelular de calcio y se activan cinasas que conllevan a una disminución del umbral de descarga y potenciación de las corrientes de sodio. El estímulo repetitivo reduce el umbral de activación de los nociceptores, con un aumento en su respuesta ante cualquier estímulo que, incluso, puede llegar a generarse espontáneamente [46,47].

La inflamación neurogénica (vasodilatación y edema) está mediada por péptidos vasoactivos relacionados con el gen de la calcitonina (CGRP), la sustancia P y la neurocinina A, que también promueven la sensibilización y la disminución del umbral de despolarización. Este mecanismo se extiende a los tejidos sanos circundantes (Fig. 4).

\section{Resiembra nerviosa y sinapsis efápticas}

Después de la lesión de un nervio, se generan puntos activos neuronales (neuromas) con actividad espontánea y evocada que producen estímulos sostenidos hacia la médula espinal. Esta alteración funcional del estímulo nervioso en los axones lesionados es interpretada como dolorosa por el soma neuronal del aferente primario en el ganglio de la raíz dorsal. Desde aquí, se activa una regeneración por medio de sustancias neurotróficas, que da lugar a nuevas terminaciones nerviosas con receptores $\alpha$-adrenérgicos (de tipo simpático) y a canales de sodio y de calcio dependientes de voltaje, inmaduros en su mayoría, con actividad independiente, espontánea y permanente $[43,45]$. Además, los axones del sistema nervioso simpático se proyectan hacia las neuronas del ganglio de la raíz dorsal, como sucede en el llamado 'dolor mantenido por el simpático'. Al componente simpático lo relevan fibras A- $\beta$, en reemplazo de las fibras $\mathrm{C}$, que asumen funciones para las cuales no se diseñaron, lo que produce una alteración en la interpretación de los estímulos activadores y, como consecuencia, la perpetuación del dolor $[43,45,47]$.

\section{Generación de descargas ectópicas}

Los factores neurotróficos NGF y GDNF mantienen y nutren las fibras nerviosas por mecanismos de transporte retrógrado. Cuando se alteran estos mecanismos, las neuronas sensitivas presentan cambios en su expresión génica, como aumento en la cantidad de receptores y activadores; consecuentemente, se modifica la expresión de los canales de sodio dependientes de voltaje, lo que facilita la propagación de los estímulos dolorosos [43,51-54] (Fig. 5).

\section{Sensibilización central}

La descarga sostenida de las fibras C libera presinápticamente sustancias neuroexcitatorias como glutamato, neurocinina y 
sustancia $\mathrm{P}$, con lo que se activan las neuronas de las láminas I, II y V de Rexed del asta dorsal por un fenómeno denominado wind-up. En consecuencia, se activan receptores AMPA y NMDA [46-48].

La retirada del magnesio del receptor, activado por glutamato, permite la entrada masiva de calcio en la segunda neurona y mantiene la activación del potencial de acción coadyuvado por segundos y terceros mensajeros ( $c$-fos y c-jun) por la vía del monóxido de nitrógeno y, como consecuencia, genera una ampliación del campo receptivo del estímulo inicial periféricamente, una disminución del umbral de descarga celular y cambios en la expresión génica del ARN mensajero; ello altera la respuesta normal ante cualquier tipo de estímulo, como sucede en la alodinia [46-48] (Fig. 6).

\section{Reorganización central de fibras}

Las lesiones de los nervios periféricos inducen procesos de neuroplasticidad. Las fibras A- $\beta$, que terminan en las láminas III y IV, estimuladas por el factor de crecimiento neuronal, se proyectan a la lámina II, que, en condiciones normales, sólo recibe fibras nociceptivas de tipo C. Esta variación se relaciona con la aparición de dolor producido por estímulos no nocivos (alodinia) $[43,45,46]$ (Fig. 7).

\section{Desinhibición central}

Es la pérdida de controles inhibitorios por la muerte de interneuronas del asta dorsal y por disminución del control superior de la vía descendente. En consecuencia, se produce una disminución del número de receptores que responden al GABA, a la glicina y a los opioides. También se produce una alteración en la sinapsis de las células inhibitorias, que actúan sobre los aferentes de primer y segundo orden y dejan libre el paso de estímulos dolorosos, que se perciben con mayor intensidad $[45,48]$.

\section{Correlación de los síntomas y signos con los mecanismos}

Estos cambios se manifiestan con síntomas y signos variados, que pueden ser espontáneos o evocados. Las relaciones entre los síntomas, los signos y los mecanismos descritos pueden entrecruzarse y configurar un cuadro clínico que se conoce como complejo sindrómico neuropático $[45,55]$. Los síntomas y signos pueden cla-
Tabla VIII. Niveles de evidencia de anticonvulsionantes.

\begin{tabular}{|c|c|c|c|}
\hline Autor & Objetivo & Calificación & Observaciones \\
\hline Mellegers & $\begin{array}{l}\text { Gabapentina para } \\
\text { dolor neuropático } \\
\text { Revisión sistemática para } \\
\text { literatura metaanalítica }\end{array}$ & $\begin{array}{l}\text { Evidencia 1+ } \\
\text { Recomendación B }\end{array}$ & RR: $2,5(1,9-3,4)$ \\
\hline McQuay et al & $\begin{array}{l}\text { Anticonvulsionantes } \\
\text { en dolor agudo y crónico } \\
\text { Revisión sistemática } \\
\text { metaanalítica } \\
\text { Excluyen la migraña } \\
\text { y las cefaleas }\end{array}$ & $\begin{array}{l}\text { Evidencia } 1++ \\
\text { Recomendación B }\end{array}$ & $\begin{array}{l}\text { Neuralgia del trigémino } \\
\text { Carbamacepina RR } 2,5(2,0-3,4) \\
\text { Neuralgia posherpética gabapentina } \\
\text { NNT } 3,2(2,4-5,0) \\
\text { Neuropatía diabética NNT } 3,2(2,4-5,0) \text {, } \\
\text { carbamacepina NNH } 3,7(2,4-7,8) \\
\text { Gabapentina NNH 2,5 }(2,0-3,2) \\
\text { Fenitoína NNH 3,2 }(2,1-6,3)\end{array}$ \\
\hline Fondey & $\begin{array}{l}\text { Gabapentina. Síndrome } \\
\text { de Guillain-Barré }\end{array}$ & $\begin{array}{l}\text { Evidencia } 1++ \\
\text { Recomendación B }\end{array}$ & Dosis únicas \\
\hline Morello & $\begin{array}{l}\text { Gabapentina } \\
\text { frente a amitriptilina }\end{array}$ & $\begin{array}{l}\text { Evidencia } 1++ \\
\text { Recomendación B }\end{array}$ & No se encontraron diferencias \\
\hline Serpell & $\begin{array}{l}\text { Gabapentina DN } \\
\text { frente a placebo } \\
2.400 \mathrm{mg}\end{array}$ & $\begin{array}{l}\text { Evidencia } 1+ \\
\text { Recomendación B }\end{array}$ & Posible cointervención \\
\hline McCleane & Lamotrigina & $\begin{array}{l}\text { Evidencia 1+ } \\
\text { Recomendación B }\end{array}$ & $\begin{array}{l}\text { Diferentes dolores, en ninguno } \\
\text { mejoró el dolor más del } 50 \%\end{array}$ \\
\hline Ric & $\begin{array}{l}\text { Gabapentina NPH } \\
\text { frente a placebo }\end{array}$ & $\begin{array}{l}\text { Evidencia } 1+ \\
\text { Recomendación B }\end{array}$ & \\
\hline
\end{tabular}

Tabla IX. Niveles de evidencia de los opiáceos.

\begin{tabular}{|c|c|c|c|}
\hline Autor & Objetivo & Calificación & Observaciones \\
\hline Dellemijn & Fentanil intravenoso & $\begin{array}{l}\text { Evidencia } 1+ \\
\text { Recomendación B }\end{array}$ & $\begin{array}{l}\text { Más efectivo para el dolor nociceptivo } \\
\text { que para el dolor neuropático }\end{array}$ \\
\hline Sidallal & $\begin{array}{l}\text { Morfina intratecal, } \\
\text { morfina y clonidina }\end{array}$ & $\begin{array}{l}\text { Evidencia 1- } \\
\text { Recomendación C }\end{array}$ & Resultados no concluyentes \\
\hline Raja & Opiáceos & $\begin{array}{l}\text { Evidencia 1- } \\
\text { Recomendación C }\end{array}$ & Resultados no concluyentes \\
\hline Benedetis & $\begin{array}{l}\text { Opiáceos en DN } \\
\text { y dolor nociceptivo } \\
\text { (bupremorfina) }\end{array}$ & $\begin{array}{l}\text { Evidencia 1- } \\
\text { Recomendación C }\end{array}$ & Resultados no concluyentes \\
\hline Sindrup & $\begin{array}{l}\text { Tramadol, alodinia } \\
\text { en dolor neuropático }\end{array}$ & $\begin{array}{l}\text { Evidencia } 1+ \\
\text { Recomendación C }\end{array}$ & Serios efectos secundarios \\
\hline Watson & $\begin{array}{l}\text { Oxicodona en } \\
\text { dolor neuropático }\end{array}$ & $\begin{array}{l}\text { Evidencia } 1+ \\
\text { Recomendación C }\end{array}$ & \\
\hline
\end{tabular}

Tabla X. Niveles de evidencia de los anestésicos locales y la ketamina.

\begin{tabular}{llll}
\hline Autor & Objetivo & Calificación & Observaciones \\
\hline Attal & $\begin{array}{l}\text { Lidocaína. } \\
\text { Dolor central }\end{array}$ & $\begin{array}{l}\text { Evidencia 1- } \\
\text { Recomendación C }\end{array}$ & Pérdida del ciego \\
\hline Haines & $\begin{array}{l}\text { Ketamina frente a } \\
\text { placebo en dolor central }\end{array}$ & $\begin{array}{l}\text { Evidencia 1+ } \\
\text { Recomendación C }\end{array}$ & Se suspende por efectos adversos \\
\hline Mercadante & $\begin{array}{l}\text { Ketamina en pacientes } \\
\text { con cáncer en trata- } \\
\text { miento con morfina }\end{array}$ & $\begin{array}{l}\text { Evidencia 1+ } \\
\text { Recomendación C }\end{array}$ & $\begin{array}{l}\text { Efectivo, pero hay evidencias de } \\
\text { que presenta efectos adversos }\end{array}$ \\
& & & \\
\hline
\end{tabular}




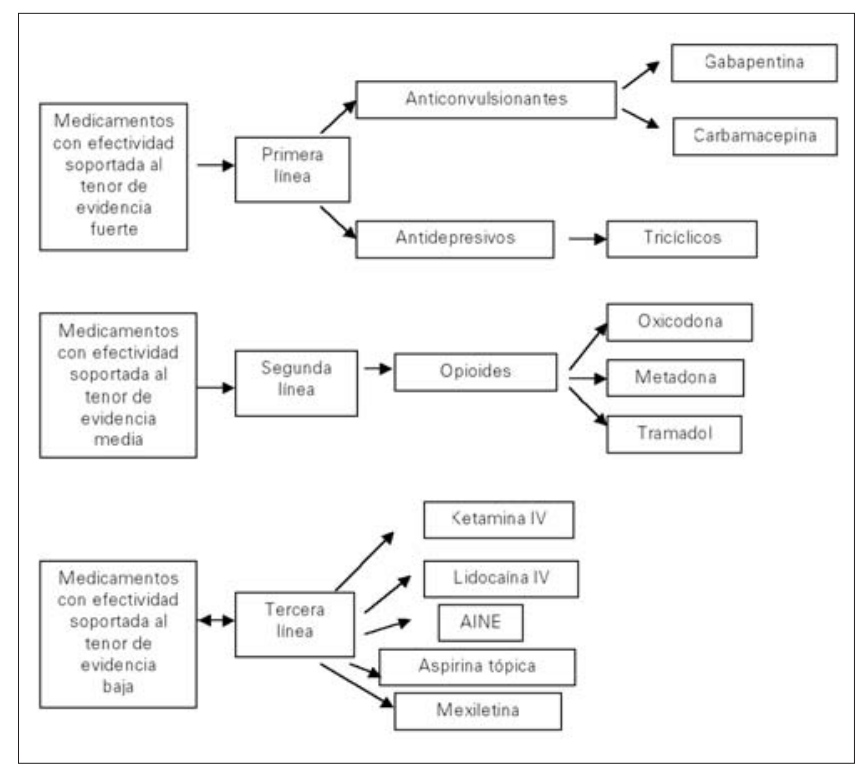

Figura 8. Tratamiento farmacológico del dolor neuropático.

La presentación espontánea de síntomas y signos se relaciona con la actividad persistente de receptores activadores. La presentación evocada implica sensibilización de los mismos. Estas extrapolaciones se derivan de lesiones neurológicas inducidas en estudios experimentales [43-45,54] (Tabla V).

\section{TRATAMIENTO}

El DN no tiene un tratamiento único y absoluto. Los mejores acercamientos terapéuticos han logrado una mejoría hasta en un $30 \%$ de los casos [56]. Estos resultados tienen como base estudios clínicos de observación, experiencias anecdóticas y hallazgos en modelos experimentales. La evidencia no ha comunicado esquemas de tratamiento que solucionen este problema, que afecta al $5 \%$ de la población [57].

Las presentes guías hacen un análisis de las intervenciones farmacológicas dentro de un marco convencional. Se incluyeron los grupos farmacológicos que generalmente utilizan quienes tratan el DN de manera general o específica. No se incluyen propuestas de la medicina tradicional y complementaria, a la espera de hallar conectores que permiten relacionarla de manera objetiva con las intervenciones farmacológicas -su impacto desborda el marco técnico y el rigor académico-.

\section{Antidepresivos}

Los antidepresivos tricíclicos tienen acciones similares a los anticonvulsionantes en el tratamiento del DN. No se diferencian de manera específica las acciones que los cualifican entre sí. A todos se les confiere una efectividad similar $-50 \%$ de mejoría del dolor, en comparación con el placebo; número necesario a tratar (NNT): 2; efectos adversos menores al 30\%- [58].

La imipramina, comparada con la codeína, muestra una evidencia significativa en el alivio del DN provocado de manera experimental, al parecer por inhibición del mecanismo de sumación temporal del dolor [59]. Esto permite extrapolar efectos positivos clínicamente.

El metaanálisis de McQuay et al confiere a los antidepresivos tricíclicos una evidencia significativa para el tratamiento de la neuropatía diabética, la neuralgia postherpética y el DN facial
Tabla XI. Niveles de evidencia de varios medicamentos.

\begin{tabular}{|c|c|c|c|}
\hline Autor & Objetivo & Calificación & Observaciones \\
\hline Byas & $\begin{array}{l}\text { Clonidina frente } \\
\text { a placebo NDD }\end{array}$ & $\begin{array}{l}\text { Evidencia 1- } \\
\text { Recomendación C }\end{array}$ & Sesgo \\
\hline McClaude & $\begin{array}{l}\text { Pancreocimina } \\
\text { coadyuvante } \\
\text { de opioides }\end{array}$ & $\begin{array}{l}\text { Evidencia } 1++ \\
\text { Recomendación A }\end{array}$ & Falta de eficacia \\
\hline Minoti & $\begin{array}{l}\text { Diclofenaco/ } \\
\text { codeína/ } \\
\text { imipramina }\end{array}$ & $\begin{array}{l}\text { Evidencia } 1++ \\
\text { Recomendación A }\end{array}$ & $\begin{array}{l}\text { Falta de eficacia. } \\
\text { No hay diferencias. } \\
\text { Efectos adversos }\end{array}$ \\
\hline Nicolajsen & $\begin{array}{l}\text { Mematine DM } \\
\text { POP amputación }\end{array}$ & $\begin{array}{l}\text { Evidencia } 1+ \\
\text { Recomendación A }\end{array}$ & Falta de eficacia \\
\hline
\end{tabular}

atípico [58] (Tabla VI). No hay diferencias significativas entre fármacos de este grupo, excepto porque la paroxetina y la mianserina son menos efectivas que la imipramina.

Con evidencias sólidas, Kingery [57] señala a los antidepresivos tricíclicos como efectivos en el tratamiento de las polineuropatías inespecíficas (Tabla VII).

\section{Anticonvulsionantes}

El metaanálisis acerca del tratamiento de DN periférico elaborado por Kingery, junto con la Colaboración Cochrane [57], respalda con una evidencia significativa la efectividad de la carbamacepina en la neuralgia del trigémino y la eficacia de la gabapentina en el tratamiento de la neuralgia postherpética y de otras neuropatías periféricas (Tabla VII). Para el tratamiento de la neuropatía diabética, la evidencia respalda utilizar tanto la carbamacepina como la gabapentina [60] (Tabla VIII). La acción de la fenitoína no es concluyente en el mismo estudio y muestra resultados conflictivos en el tratamiento de las polineuropatías [57].

Serpell [61] encuentra evidencias de que la gabapentina reduce el dolor y mejora la calidad de vida de las personas que sufren síndromes dolorosos neuropáticos de diferentes etiologías (Tabla VIII).

Con buena evidencia, pero de manera no concluyente por falta de tamaño de la muestra, Morello y McCleane et al demuestran que la amitriptilina y la gabapentina alivian de manera similar el dolor de la neuropatía diabética [62,63] (Tabla VIII). Aunque los resultados se inclinaron en contra de la amitriptilina por sus efectos adversos y en contra de la gabapentina por los costos, los autores sugieren desarrollar estudios con mayor tamaño muestral.

Harke et al señalan que la carbamacepina es eficaz de manera significativa en el tratamiento del DN con dosis superiores a 600 mg/día [64]. Kingery, con una evidencia sólida, califica la carbamacepina como efectiva en el tratamiento de polineuropatías inespecíficas [57].

Pandey et al [65] demostraron que la gabapentina mejora el dolor de características neuropáticas en el síndrome de Guillain-Barré [65]. La acción de la gabapentina en el alivio de la neuralgia postherpética se respalda con fuerza de evidencia en el estudio de Rice y Maton [66].

\section{Opioides}

Según el estudio de Harke et al, entre 60 y 90 mg/día de morfina de liberación sostenida no mejoran el dolor de características neuropáticas [64]. 


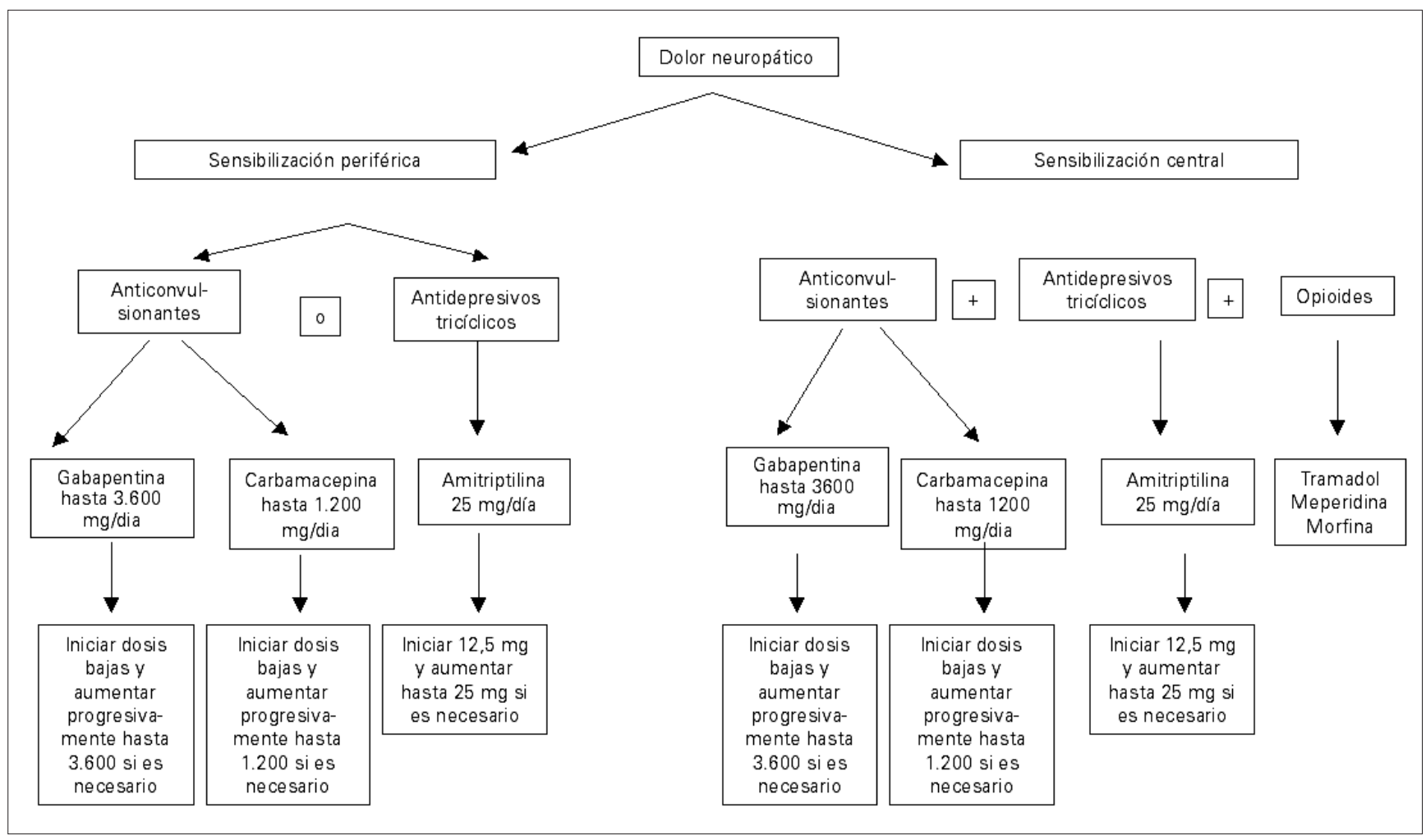

Figura 9. Flujograma de tratamiento propuesto por el equipo investigador. El orden propuesto no descarta la combinación de medicamentos. Las dosis de cada uno de los medicamentos propuestos se debe contextualizar dentro de intervalos terapéuticos. Variables como la edad, las condiciones del paciente o la etiología, no están apoyadas por un marco de evidencia. Los estudios no respaldan con fuerza la evidencia para el tratamiento en población infantil.

Kingery [57] demuestra que la morfina endovenosa tiene efectos contradictorios en el tratamiento de neuropatías dolorosas. De igual forma, este autor señala a la codeína como inefectiva en el tratamiento del DN [57].

Sindrup et al concluyen, con evidencia significativa, que el tramadol disminuye la alodinia en la neuropatía de etiología variada [67]. El estudio de Watson y Pharm indica que la oxicodona es efectiva como analgésico en el tratamiento de la neuralgia postherpética [68] (Tabla IX).

\section{Analgésicos antiinflamatorios no esteroideos (AINE)}

Con una evidencia satisfactoria, Kingery demuestra que los AINE tienen efectos débiles en el tratamiento de las polineuropatías dolorosas [57].

\section{Otros}

La ketamina demostró tener un efecto favorable para el DN refractario; sin embargo, los efectos colaterales limitan su uso y su recomendación [55]. Con las mismas restricciones, la ketamina fue efectiva en pacientes con $\mathrm{DN}$ de origen oncológico refractario a la morfina $[69,70]$ (Tabla $X)$.

La evidencia también respalda la acción favorable de la ketamina y la lidocaína endovenosas en el tratamiento de las polineuropatías dolorosas [57]. De igual forma, se restringe la acción de la clonidina endovenosa, de la lidocaína epidural o tópica, así como de la mexiletina y la capsaicina, que evidencian efectos contradictorios en el tratamiento de estos cuadros dolorosos [57] (Tabla XI).
Kingery indica que el magnesio, el propanolol, el loracepam y la fentolamina son efectivos en el tratamiento del DN [57]. Para el tratamiento del síndrome regional complejo, los corticoides orales fueron efectivos, pero no lo fueron el dimetil sulfóxido tópico, la clonidina epidural, el bretilio, la ketancerina, la guanatedina, la reserpina, el droperidol y la atropina. La fentolamina endovenosa evidenció resultados contradictorios [57] (Tabla VII).

\section{CONCLUSIONES}

Con base en el análisis y en las tendencias de los resultados en el tratamiento del DN, se pueden hacer las siguientes inferencias:

- No existe un esquema específico para el tratamiento del dolor neuropático.

- Escasean los estudios que relacionen de manera específica el tratamiento del DN con los mecanismos fisiopatológicos que lo generan.

- Los antidepresivos y los anticonvulsionantes constituyen la piedra angular del tratamiento del DN.

- No existe una evidencia contundente de que los opioides y los AINE sirvan para el DN.

- Es necesario realizar estudios que utilicen combinaciones de medicamentos que, eventualmente, demuestren mejores resultados en el tratamiento del DN.

- No existe evidencia que respalde el tratamiento interdisciplinario del DN, como sí lo hay con referencia al tratamiento del dolor crónico. 


\section{RECOMENDACIONES}

Como resultado de este análisis, y sin pretender presentar un algoritmo específico para el tratamiento del DN, se propone una escalera descendente de efectividad estructurada sobre un esquema definido de medicamentos de primera, segunda y tercera línea. Esto debe ponerse en relación con la clínica y, naturalmente, con las variables que cualifican la intervención individual en cada paciente (Figs. 8 y 9).

\section{GLOSARIO DE TÉRMINOS}

- Alodinia mecánica: es la percepción de dolor a partir de un estímulo mecánico no doloroso; por ejemplo, después de exponer la región afectada a una corriente de aire.

- Alodinia térmica: es la sensación anormal de dolor a partir de un estímulo no nocivo con calor o frío.

- Disestesia: es una sensación anormal descrita como incómoda por el paciente. Igual que la parestesia, puede ser espontánea o provocada en el examen físico; cuando el paciente la refiere como dolorosa, puede considerarse una alodinia.

- Dolor referido: es el que se siente en otra área diferente al tejido afectado. El dolor se refiere de acuerdo al dermatoma, miotoma o esclerotoma.

- Hiperalgesia: es una respuesta dolorosa exagerada a un estímulo algogénico. Puede ser térmica o mecánica, según el tipo de estímulo.

- Hiperpatía: es la respuesta anormalmente dolorosa a un estímulo o la reacción exagerada a un estímulo repetitivo.

- Parestesia: es una sensación anormal descrita como no dolorosa. Puede ser espontánea o provocada en el examen físico por un estímulo doloroso o no.

- Postsensación: es la persistencia anormal de una sensación causada por un estímulo después de que éste haya terminado.

- Sumación: es la sensación dolorosa que se incrementa con el estímulo repetido, aunque éste permanezca constante.

\section{BIBLIOGRAFÍA}

1. Howard LF, Hill RG. Neurophatic pain: the near and far horizon. In Hansson PT, Fields HL, Hill RG. Marchettini P, eds. Neuropathic pain; pathophysiology and treatment. Seattle: IASP Press; 2001. p. 251-2.

2. Costigan M, Woolf CJ. Pain: molecular mechanisms. J Pain 2000; 1 (Suppl 1): 35-44.

3. Buonocore M, Bonezzi C. Neuropathic pain syndromes: from their diagnosis to the return to work. Proposal of a model for rapid evaluation and therapy based on their pathogenic mechanisms. G Ital Med Lav Ergon 2001; 23: 484-93.

4. Hansson P, Lacerenza M, Marchettini P. Aspects of clinical and experimental neuropathic pain: the clinical perspective. In Hansson PT, Fields HL, Hill RG, Marchettini P, eds. Neuropathic pain; pathophysiology and treatment. Seattle: IASP Press; 2001. p. 1-18.

5. Bennett M. The LANSS pain scale: the Leeds assessment of neuropathic symptoms and signs. Pain 2001; 92: 147-57.

6. Zakrzewska JM. Diagnosis and differential diagnosis of trigeminal neuralgia. Clin J Pain 2002; 18: 14-21.

7. Schmader KE. Epidemiology and impact on quality of life of postherpetic neuralgia and painful diabetic neuropathy. Clin J Pain 2002; 18: 350-4.

8. Browsher D. The lifetime occurrence of herpes zoster and prevalence of post herpetic neuralgia: A retrospective survey in an elderly population. Eur J Pain 1999; 3: 335-42.

9. Harden N, Cohen M. Neuropathic pain: from mechanisms to treatment strategies. Unmet needs in the management of neuropathic pain. J Pain Symptom Manage 2003; 25: $12-7$.

10. Feder G, Eccles M, Grol R, Griffiths C, Grimshaw J. Using clinical guidelines. BMJ 1999; 318: 720-30.

11. Shekelle PG, Woolf SH, Eccles M, Grimshaw J. Clinical guidelines. Developing guidelines. BMJ 1999; 318: 593-6.

12. Guyatt GH, Sackett DL, Sinclair JC, Hayward R, Cook DJ, Cook RJ. Users' guides to the medical literature. IX. A method for grading health care recommendations. Evidence-Based Medicine Working Group. JAMA 1995; 274: 1800-4

13. Rodney J, Gene J. Guidelines for clinical guidelines. A simple, pragmatic strategy for guideline development. BMJ 1998; 317: 427-8,

14. Eccles M, Freemantle N, Mason J. North of England evidence based guidelines development project: methods of developing guidelines for efficient drug use in primary care. BMJ 1998; 316: 1232-5.

15. Smith R. Conflict of interest and the BMJ. BMJ 1994; 308: 4.

16. Sandkuhler J, Bromm B, Gebhart GF. Progress in brain research. Pain 2000; 129 .

17. Giamberardino MA. Update review: refresher course syllabus. Seattle: IASP Press; 2002.

18. Merskey H, Bogduk N. Classification of chronic pain. Description of chronic pain syndromes and definitions of pain terms. 2 ed. Seattle: IASP Press, 1994.

19. Backonja MM. Defining neuropathic pain. Anesth Analg 2003; 97 : 785-90.

20. Carter GT, Galer BS. Advances in the management of neuropathic pain. Phys Med Rehabil Clin North Am 2001; 12: 447-59.
21. Hansson P. Neuropathic pain: Clinical characteristics and diagnostic workup. Eur J Pain 2002; 6 (Suppl): 47-50.

22. Dworkin RH, Backonja M, Rowbotham MC, Allen RR, Argoff CR, Bennett GJ, et al. Advances in neuropathic pain. Diagnosis, mechanisms and treatment recommendations. Arch Neurol 2003; 60: 1524-34.

23. Boureau F, Doubrére JF, Luu M. Study of verbal description in neuropathic pain. Pain 1990; 42: 145-52.

24. Hall TM, Elvery RL. Nerve trunk pain: physical diagnosis and treatment. Man Ther 1999; 4: 63-73.

25. Wasner G, Schattschneider J, Binder A, Baron R. Complex regional pain syndrome-diagnostic, mechanisms, CNS involvement and therapy. Spinal Cord 2003; 41: 61-75.

26. Hagen NA. Reproducing a cancer patient's pain on physical examination: bedside provocative maneuvers. J Pain Symptom Management 1999; 18: 406-11.

27. Wang HS. Neurobehavioral outcome of infants sonographic lenticulostriate vasculopathy (letter). Pediatr Neurol 2001; 24.

28. Lee D, Van Holsbeeck MT, Janevski PK, Ganos DL, Ditmars DM, Darian VB. Diagnosis of carpal tunnel syndrome. Ultrasound versus electromyography. Radiol Clin North Am 1999; 37: 860-72.

29. Zúñiga JR, Meyer RA, Gregg JM, Miloro M, Davis LF. The accuracy of clinical neurosensory testing for nerve injury diagnosis. J Oral Maxillofac Surg 1998; 56: 2-8.

30. Logigian EL, Steere AC. Clinical and electrophysiologic findings in chronic neuropathy of Lyme disease. Neurology 1992; 2: 303-11.

31. Bose KK, Chakraborty J, Khuder S, Smith-Mensah WH, Robinson J. Lysyl oxidase activity in the cells of flexor retinaculum of individuals with carpal tunnel syndrome. J Occup Environ Med 2000; 42: 582-7.

32. Jordan SE, Machleder HI. Diagnosis of thoracic outlet syndrome using electrophysiologically guided anterior scalene blocks. Ann Vasc Surg 1998; 12: 260-4.

33. Backonja MM, Galer BS. Pain assessment and evaluation of patients who have neuropathic pain. Neurol Clin 1998; 16: 775-89.

34. Chong MS, Bajwa ZH. Diagnosis and treatment of neuropathic pain. J Pain Symptom Manage 2003; 25: 4-11.

35. Dellemijn PLI, Fields HL, Allen RR, Mckay WR, Rowbotham MC. The interpretation of pain relief and sensory changes following sympathetic blockade. Brain 1994; 117: 1475-87.

36. Padua L, Padua R, Monaco M, Aprile I, Tonali P. Multiperspective assessment of carpal tunnel syndrome: A multicenter study. Neurology 1999; 53: 1654-9.

37. Kupfer DM, Bronson J, Lee GW, Beck J, Gillet J. Differential latency testing: a more sensitive test for radial tunnel syndrome. J Hand Surg Am 1998; 23: 859-64. Erratum in: J Hand Surg Am 1999; 24: 421.

38. Leffler CT, Gozani SN, Cros D. Median neuropathy at the wrist: Diagnostic utility of clinical findings and an automated electrodiagnostic device. J Occupational Environ Med 2000; 42: 398-409.

39. Chang MH, Wei SJ, Chiang HL, Wang HM, Hsieh PF, Huang SY. Comparison of motor conduction techniques in the diagnosis of carpal tunnel syndrome. Neurology 2002; 58: 1603-7.

40. Gentilello LM. Near-infrared spectroscopy versus compartment pres- 
sure for the diagnosis of lower extremity compartmental syndrome using electromyography-determined measurements of neuromuscular function J Trauma 2001; 51: 1-8.

41. Cornblath DR, Chaudhry V, Carter K, Lee D, Seysedadr M, Joh T. Total neuropathy socre: validation and reliability study. Neurology 1999; 53: 1660-4.

42. Ambroz C, Scott A, Ambroz A, Talbott EO. Chronic low back pain assessment using surface electromyography. J Occup Environ Med 2000; 42: 660-9

43. Bridges D, Thompson SWN, Rice ASC. Mechanisms of neuropathic pain. Bri J Anaesth 2001; 87: 12-26.

44. Jensen TS, Baron R. Translation of symptoms and signs into mechanisms in neuropathic pain. Pain 2003; 102: 1-8.

45. Woolf CJ, Mannion RJ. Neuropathic Pain: aetiology, symptoms, mechanisms, and management. Lancet 1999; 353: 1959-64.

46. Bolay H, Moskowitz MA. Mechanisms of pain modulation in chronic syndromes. Neurology 2002; 59 (Suppl 2): 2-7.

47. Schwartzman RJ, Grothusen J, Kiefer TR, Rohr P. Neuropathic central pain. Epidemiology, etiology and treatment options. Arch Neurol 2001; 58: 1547-50.

48. Costigan M, Wolf CJ. Pain: molecular mechanisms. J Pain 2000; 1 (Suppl 1): 35-44.

49. Wool C, Salter M. Neuronal plasticity: increasing the gain in pain. Science 2000; 288: 1765-8.

50. Besson JM. The neurobiology of pain. Lancet 1999; 353: 1610-5.

51. Black JA, Cummins TR, Plumptom C, Chen YH, Hormuzdiar W, Clare JJ, et al. Upregulation of a silent sodium channel after peripheral, but not central, nerve injury in DRG neurons. J Neurophysiol 1999; 82: $2776-85$.

52. Waxman SG. The molecular pathophysiology of pain: abnormal expression of sodium channel genes and its contributions to hyperexcitability of primary sensory neurons. Pain 1999; (Suppl 6): 133-40.

53. Rowbotham M. Neuropathic pain: from basic science to evidencebased treatment. Pain 2002; an updated review, refresher course syllabus. Seattle: IASP Press; 2002. p. 165-76.

54. Wallace MS. Emerging drugs for neuropathic pain. Expert Opin Emerging Drugs 2001; 6: 249-59.

55. Woolf CJ, Max MB. Mechanism-based pain diagnosis. Anesthesiology 2001; 95: 241-9.

56. Farrar JT, Portenoy RK, Berlin JA, Kinman JL, Strom BL. Defining the clinically important difference in pain outcome measures. Pain 2000; 88: 287-94.

57. Kingery WS. A critical review of controlled clinical trials for peripher- al neuropathic pain and complex regional pain syndromes. Pain 1997; 73: $123-39$.

58. McQuay HJ, Tramer M, Nye BA, Carroll D, Wiffen PJ, Moore RA. A systematic review of antidepressants in neuropathic pain. Pain 1996; 68: 217-27.

59. Enggaard TP, Poulsen L, Arendt-Nielsen L, Hansen SH, Bjornsdottir I, Gram LF, et al. The analgesic effect of codeine as compared to imipramine in different human experimental pain models. Pain 2001; 92: $277-82$.

60. Collins SL, Moore A, McQuay HJ, Wiffen P. Antidepressants and anticonvulsants for diabetic neuropathy and postherpetic neuralgia: a quantitative systematic review. J Pain Symptom Manage 2000; 20: 449-58.

61. Serpell MG. Gabapentin in neuropathic pain syndromes: a randomised, double-blind, placebo-controlled trial. Pain 2002; 99: 557-66.

62. Morello CM, Leckband SG, Stoner CP, Moorhouse DF, Sahagian GA. Randomized double-blind study comparing the efficacy of gabapentin with amitriptyline on diabetic peripheral neuropathy pain. Arch Intern Med. 1999; 159 16: 1931-7.

63. McCleane GJ. Comment on: Serpell et al, gabapentin in neuropathic pain syndromes: a randomised double-blind, placebo controlled trial [author reply 228]. Pain 2003; 103: 227.

64. Harke H, Gretenkort P, Ladleif HU, Rahman S, Harke O. The response of neuropathic pain and pain in complex regional pain syndrome I to carbamazepine and sustained-release morphine in patients pretreated with spinal cord stimulation: a double blinded randomized study. Anesth Analg 2001; 92: 488-95.

65. Pandey C, Neeta B, Garima G, Namita S, Arvind B, et al. Gabapentin for the treatment of pain in Guillain-Barre syndrome. A double-blinded, placebo-controlled, crossover study. Anest Analg 2002; 95: 1719-23.

66. Rice ASC, Maton S. Gabapentin in postherpetic neuralgia: a randomised, double blind, placebo controlled study. Pain 2001; 94: 215-24.

67. Sindrup SH, Hesen TS. Efficacy of pharmacological treatments of neuropathic pain: an update and effect related to mechanism of drug action. Pain 1999; 83: 389-400.

68. Watson CPN, Pharm NB. In PP. Efficacy of oxycodone in neuropathic pain. A randomised trial in postherpetic neuralgia. Neurology 1998; 50: $1837-41$.

69. Haines DR, Gaines SP. N of 1 randomised controlled trials of oral ketamine in patients with chronic pain. Pain 1999; 83: 283-7.

70. Mercadante S, Arcuri E, Tirelli W, Casuccio A. Analgesic effect of intravenous ketamine in cancer patients on morphine therapy: a randomized, controlled, double-blind, crossover, double-dose study. J Pain Symptom Manage 2000; 20: 246-52.

\section{GUÍAS DE PRÁCTICA CLÍNICA DEL DOLOR NEUROPÁTICO (II)}

Resumen. Introducción. El dolor neuropático $(D N)$ se presenta hasta en el $5 \%$ de la población. Después de una búsqueda bibliográfica en varias bases de datos, se determinó que hasta la fecha no existen protocolos que orienten al médico que no es especialista en dolor para tratar el DN y mejorar la calidad de vida de los pacientes. Objetivo. Presentar unas guías de tratamiento del DN dirigidas a médicos no especialista en dolor de habla hispana. Se realizó una búsqueda bibliográfica para fundamentar los resultados y las conclusiones con la metodología de la medicina basada en la evidencia. Desarrollo. En primera instancia se revisan los métodos clínicos y paraclínicos más eficaces para el diagnóstico del DN y se describe la escala de LANSS como la opción más adecuada para la evaluación clínica del DN. Posteriormente, se describen las vías anatómicas y la fisiología del dolor y se revisan las variables moleculares involucradas. Finalmente, se señalan las opciones terapéuticas actuales y se propone un algoritmo de tratamiento del DN. Conclusiones. No existe un esquema específico para el tratamiento del DN. Actualmente, los antidepresivos y los anticonvulsionantes son la piedra angular del tratamiento. Se requieren ensayos clínicos que evidencien la eficacia del uso de medicamentos combinados. [REV NEUROL 2005; 40: 303-16]

Palabras clave. Diagnosis. Dolor neuropático. Ensayos clínicos. Fisiología. Metaanálisis. Tratamiento.

\section{GUIAS DE PRÁTICA CLÍNICA DA DOR NEUROPÁTICA (II)}

Resumo. Introdução. A dor neuropática (DN) regista-se em $5 \%$ da população. Após uma pesquisa bibliográfica em várias bases de dados, concluiu-se que até à data não existiam protocolos que orientassem o médico não especialista na dor, para o tratamento da DN e melhoria da qualidade de vida dos doentes. Objectivo. Apresentar guias de tratamento da DN dirigidas a médicos não especialistas na dor, em língua espanhola. Realizou-se uma pesquisa bibliográfica para fundamentar os resultados e conclusões com a metodologia da medicina baseada na evidencia. Desenvolvimento. Em primeira instância fez-se a revisão dos métodos clínicos e paraclínicos mais eficazes para o diagnóstico da DN e descreveuse a escala de LANSS como a opção mais adequada para a avaliação clínica da DN. Posteriormente, descreveram-se as vias anatómicas e a fisiologia da dor, e fez-se a revisão das variáveis moleculares envolvidas. Finalmente, assinalaram-se as opções terapêuticas actuais e propôs-se um algoritmo de tratamento da DN. Conclusões. Não existe um esquema específico para o tratamento da $D N$. Actualmente, os antidepressivos e os anticonvulsivantes são a pedra angular do tratamento. É necessário realizarem-se ensaios clínicos que evidenciem a eficácia do uso de medicamentos combinados. [REV NEUROL 2005; 40: 303-16]

Palavras chave. Diagnóstico. Dor neuropática. Ensaios clínicos. Fisiologia. Meta-análise. Tratamento. 